\section{JULY 1969}

Collins wishes his colleagues, Aldrin and Armstrong, luck as they transfer to 'the Eagle'.

Some hours later, Armstrong places his foot on the lunar surface, declaring '... one small step for man, one giant leap for mankind'.

The space race era, at least the intoxicating first phase, is over.

This was rocket science in every way, a huge and complex challenge. The need for rocket science, though, isn't the exclusive province of space travel.

\section{YEARS ON}

Why is it that some ostensibly simple questions about common problems are so bafflingly difficult to answer? Two respiratory randomised controlled trials provide some insight.

Steroid treatment in preschool asthma is arguably the archetypal example: it has the prerequisite properties in abundance: refusal to let go of non-evidence based practice ('but, I've been using this method for years.' you protest), measurement of outcome subjectivity and disagreement on which end point is most important; phenotype heterogeneity, even misdiagnosis... there's almost nothing that digs heels more firmly in the ground. I could go on at length, but don't think I need to...

So, one can only applaud Alexandra Wallace and colleagues' multisite noninferiority RCT in New Zealand testing the difference in the PRAM (preschool respiratory assessment measure) 24 hours after the respective prednisolone or placebo doses. Despite a moderate degree of non-enrolment in eligible children, there were close to 500 participants.

Department of Women's and Children's Health, International Maternal and Child Health (IMCH), Uppsala University, Uppsala, Sweden; Department of Paediatrics, Länssjukhuset Gävle-Sandviken, Gävle, Sweden; Department of Child Health, Aga Khan University, Karachi, Pakistan

Correspondence to Dr Nick Brown, Department of Women's and Children's Health, International Maternal and Child Health (IMCH), Uppsala University, Uppsala 801 87, Sweden; nickjwbrown@gmail.com
The primary outcome was the change in the (well validated) Preschool Respiratory Assessment Measure (PRAM) score 24 hours after intervention. Secondary outcomes included absolute PRAM scores at 4 and 24 hours, length of emergency department and inpatient stays, admission and representation rates, time to return to normal activities and the relative proportions of children in each group given further doses of oral prednisolone or intravenous rescue medication.

There was no difference between groups for the primary outcome, the PRAM scores at 24 hours (difference between means $-0.39,95 \%$ CI -0.84 to $0.06, p=0.09$ ) being similar. Most children recovered with or without steroids within 24 hours but, the absolute PRAM score was lower in the prednisolone group at 4 and 24 hours as were admission rate, requirement for additional oral prednisolone and use of intravenous. There were no differences between groups for time taken to return to normal activities or rates of representation within 7 days.

We can't say whether the time of day of first dose is important, or season of treatment a factor ('allergy more likely in the summer, URTI triggered in the winter'), but this feels a big step forward. Some might argue that alternative primary outcomes (readmission, for example) would have been preferable, but as these are mainly dichotomous (and therefore less statistically efficient not to mention centre practice and geographical effects) might have left us with a false negative and continued angst. The rapid recovery times suggest the traditional (prime number, of course, aren't they always) 3 day course might be on the way out too, or at least that compliance with prophylaxis and inhaler technique are consistently assessed before reaching for the prescription pad. Let's see what happens. See page 339

\section{NICOTINE OR CASH?}

Though it isn't currently on the WHO top ten threats list, 'promoting' children's tobacco smoke exposure arguably should be particularly as legislative moves haven't (pun intended) stubbed it out. Mandeep Jassal and colleagues in Maryland, US, undertook an ambitious RCT targeted at children's caregivers who smoked. The implications for a decrease or even cessation are both immediate, release from an environment steeped in an enveloping yellow fug and longer term whether children, through copying their caregivers are not given a template by which the cycle of deprivation is continued for life. In the trial, standard care was augmented (intervention group) by (moderately generous) financial incentives to cut down, urinary cotinine being the measure of compliance. Counterintuitively, the incentives made no difference to cotinine derived exposure. The discussion explores potential reasons why-lack of connectivity with social networks in this group of (in this case, largely maternal) caregivers, being one potential explanation. Realistically, it seems tightening legislation is going to have to be part of any future solution. This study demonstrates why 'negative' trials are equally as important (if not more so) as 'positive' ones: we need to know which fields need to be ploughed further and which don't. See page 345

\section{SICKLE CELL DISEASE}

Sickle cell disease hasn't been a single unit 6 monthly follow-up 'entity' for a long time and Eirini Kyrana's review of recent hepatological progress demonstrates why perfectly: the liver is highly susceptible to direct and indirect damage. From sequestration to sludge to haemolysis to co-morbidities, it feels, intuitively, as if a liver review annually in these unfortunate children should be as routine as the cerebral artery doppler ultrasound, irrespective of absence of 'hard' signs. See page 315

Rocket science comes in many forms. Enjoy the rest of the issue.

ORCID iD

Nick Brown http://orcid.org/0000-0003-1789-0436 